\title{
Doxorubicin-conjugated bacteriophages carrying anti-MHC class I chain-related A for targeted cancer therapy in vitro
}

This article was published in the following Dove Press journal:

OncoTargets and Therapy

28 November 2014

Number of times this article has been viewed

\author{
Achara Phumyen ${ }^{1-3}$ \\ Siriporn Jantasorn' \\ Amonrat Jumnainsong \\ Chanvit Leelayuwat ${ }^{1-4}$ \\ 'The Centre for Research and \\ Development of Medical Diagnostic \\ Laboratories (CMDL), Faculty of \\ Associated Medical Sciences, ${ }^{2}$ The \\ Liver Fluke and Cholangiocarcinoma \\ Research Center, Faculty of \\ Medicine, ${ }^{3}$ Research Cluster: \\ Specific Health Problem of Grater \\ Maekong Subregion (SHeP-GMS), \\ ${ }^{4}$ Department of Clinical Immunology \\ and Transfusion Sciences, Faculty of \\ Associated Medical Sciences, Khon \\ Kaen University, Khon Kaen, Thailand
}

Correspondence: Chanvit Leelayuwat The Centre for Research and Development of Medical Diagnostic Laboratories (CMDL), Faculty of Associated Medical Sciences, Khon Kaen University, Khon Kaen 40002, Thailand

$\mathrm{Tel} / \mathrm{Fax}+6643202089$

Email chanvit@kku.ac.th
Background: Cancer therapy by systemic administration of anticancer drugs, besides the effectiveness shown on cancer cells, demonstrated the side effects and cytotoxicity on normal cells. The targeted drug-carrying nanoparticles may decrease the required drug concentration at the site and the distribution of drugs to normal tissues. Overexpression of major histocompatibility complex class I chain-related A (MICA) in cancer is useful as a targeted molecule for the delivery of doxorubicin to MICA-expressing cell lines.

Methods: The application of 1-ethyl-3-[3-dimethylaminopropyl] carbodiimide (EDC) chemistry was employed to conjugate the major coat protein of bacteriophages carrying anti-MICA and doxorubicin in a mildly acid condition. Doxorubicin (Dox) on phages was determined by double fluorescence of phage particles stained by M13-fluorescein isothiocyanate (FITC) and drug autofluorescence by flow cytometry. The ability of anti-MICA on phages to bind MICA after doxorubicin conjugation was evaluated by indirect enzyme-linked immunosorbent assay. One cervical cancer and four cholangiocarcinoma cell lines expressing MICA were used as models to evaluate targeting activity by cell cytotoxicity test.

Results: Flow cytometry and indirect enzyme-linked immunosorbent assay demonstrated that most of the phages ( $82 \%$ ) could be conjugated with doxorubicin, and the Dox-carrying phagedisplaying anti-MICA (Dox-phage) remained the binding activity against MICA. Dox-phage was more efficient than free drugs in killing all the cell lines tested. The half maximal inhibitory concentration $\left(\mathrm{IC}_{50}\right)$ values of Dox-phage were lower than those of free drugs at approximately 1.6-6 times depending on MICA expressions and the cell lines tested.

Conclusion: Evidently, the application of 1-ethyl-3-[3-dimethylaminopropyl] carbodiimide chemistry is effective to conjugate doxorubicin and major coat protein of bacteriophages without destroying binding activity of MICA antibodies. Dox-carrying bacteriophages targeting MICA have been successfully developed and may enable a broad range of applications in cancer-targeting chemotherapy.

Keywords: MHC class I chain-related A (MICA), phage display, doxorubicin, targeted therapy

\section{Introduction}

Over the past two decades, the therapeutic efficiency of chemotherapeutic drugs or toxins targeting tumors have been improving, which relies on the specific binding and internalization of these conjugates into the target cells. Immunoconjugates-monoclonal antibodies (mAbs) or immunoconjugates-nanoparticles coupled to highly toxic agents are becoming a significant component in anticancer treatments. ${ }^{1}$ By combining the exquisite targeting specificity of mAbs with enhanced tumor-killing power of toxic effector molecules, immunoconjugates permit sensitive discrimination between targets 
and normal tissues, resulting in reduced administration of drug concentration and fewer toxic side effects than most conventional chemotherapeutic drugs. ${ }^{2}$ Recently, a new platform of targeted anticancer therapy has been generated in the form of drug-carrying bacteriophages. ${ }^{3,4}$ This approach is based on genetically modified and chemically manipulated filamentous bacteriophages. The genetic manipulation endows the phages with the ability to display host specificity-conferring ligands. The phages are loaded with a large payload to a cytotoxic drug by chemical conjugation. Targeting of phage nanomedicines results in endocytosis, intracellular degradation, and drug release, leading to growth inhibition of the target cells in vitro with potentiation factor of more than 1,000 over the corresponding free drug. ${ }^{4}$

Natural killer group 2, member D (NKG2D) ligands show restricted expression in normal tissues, but they are frequently overexpressed in cancer and viral-infected cells. ${ }^{5}$ Many tumor cell lines and primary tumors from diverse tissue origins express NKG2D ligands such as carcinoma (lung, breast, kidney, prostate, ovary, colon, liver, and bile duct), melanoma, and some primary leukemia. ${ }^{5-12}$ The binding of NKG2D ligands to receptor activates natural killer (NK) cells and $\mathrm{T}$ cells and promotes cytotoxic lysis of the cells that express these molecules. ${ }^{13}$ Therefore, the expression of major histocompatibility complex (MHC) class I chainrelated A (MICA) on tumor cells provide us opportunities for the use of immunoconjugate drug therapy for cancer. Immunotherapy via NKG2D recognition has been exploited using chimeric antitumor $\mathrm{mAb} / \mathrm{NKG} 2 \mathrm{D}$ ligand reagents, which specifically bind to human tumor cells and enhance the lysis by NK cells in an NKG2D-dependent manner as well as elicit adaptive immune responses against cancer cells independent of NKG2D ligand expression. ${ }^{14}$ We have previously produced bacteriophages carrying mutated anti-MICA antibodies with high binding activities. ${ }^{15}$ In order to generate an effective platform for killing cancer cells, the application of 1-ethyl-3-[3-dimethylaminopropyl] carbodiimide (EDC) chemistry was employed to conjugate anticancer drug (doxorubicin) to major coat $\mathrm{g} 8 \mathrm{p}$ protein of M13 filamentous phages that carry anti-MICA antibodies. These drug-carrying phages specific to MICA antigens are more effective than free doxorubicin in killing cell lines expressing MICA.

\section{Materials and methods \\ Materials}

\section{Phage-displaying anti-MICA antibody}

Phage-displaying mutant Fab WW9B8.21 displays highaffinity anti-MICA derived from monoclonal WW9B8. ${ }^{15,16}$
These phages were used for conjugation with the anticancer drug doxorubicin· $\mathrm{HCl}$ (Enzo Life Sciences, Inc., Matford Court, Exeter, UK). All phages were amplified by infected bacteria, Escherichia coli XL1 blue (Stratagene, La Jolla, CA, USA) and rescued by VSCM13 (New England Biolabs, Inc., Ipswich, MA, USA), the helper phages, for phage stock. The stocked phages were counted and calculated as plaqueforming unit (PFU) before performing conjugation and then were stored in Tris-buffered saline (USB; Affymetrix, Inc., High Wycombe, UK)/1\% bovine serum albumin (SigmaAldrich, St Louis, MO, USA) at $4^{\circ} \mathrm{C}$.

\section{Soluble MICA antigen}

Soluble MICA (sMICA) antigen was produced from a eukaryotic expression clone carrying a full-length coding sequence of the $M I C A^{*} 009$ gene with inserted stop codons before the transmembrane portion to produce a soluble form of MICA according to a previous report. ${ }^{16}$

\section{Cell lines}

Cell lines used were a cervical cancer cell line, Hela (CCL-2; American Type Culture Collection, Manassas, VA, USA), and the cholangiocarcinoma cell lines, KKU-M055, KKU-M213, KKU-M214, KKU-M13, and KKU-MMNK1 provided by The Liver Fluke and Cholangiocarcinoma Research Center, Faculty of Medicine, Khon Kaen University, Khon Kaen, Thailand. Cells have an epithelial-like morphology growing as monolayer in Dulbecco's Modified Eagle's Medium (Thermo Fisher Scientific, Waltham, MA, USA) and HAM's F12 (Thermo Fisher Scientific) with 10\% (v/v) heat inactive fetal calf serum (PAA GmbH, Cölbe, Germany) and $100 \mu \mathrm{g} / \mathrm{mL}$ of penicillin/streptomycin sulfate (Sigma-Aldrich).

\section{Methods}

\section{Chemical conjugation of doxorubicin to phage-displaying anti-MHC class I chain-related $A$ antibodies}

The conjugation of doxorubicin to M13 bacteriophages was performed according to the previous studies. ${ }^{4,17,18}$ The phage major coat protein g8p contains three carboxylic amino acids (glu2, asp4, and asp5), which can be conjugated directly with the primary amine of doxorubicin by the application of EDC chemistry (Thermo Fisher Scientific, Waltham, MA, USA), a rapid reaction performed at mild $\mathrm{pH} 4-6 .{ }^{19}$ All conjugations were performed within a $1.5 \mathrm{~mL}$ tube. The reagents for conjugation which were the EDC solution at $\mathrm{pH}$ 4-6 were used in the reaction as the condition below. The concentration and ratio of carboxylate groups on phage particles, amine groups on doxorubicin 
molecules, and concentration of EDC were calculated at a ratio of 1 mol COOH:1 mol amine:4 mol EDC.

In the experiment, doxorubicin, phages, $0.1 \mathrm{M}$ sodium citrate buffer $\mathrm{pH} 4,5$, or 6 , and $0.75 \mathrm{NaCl}$ (Vivantis, Inc., Oceanside, CA, USA) were added into a $1.5 \mathrm{~mL}$ tube. The conjugation reaction was initiated by the addition of EDC solution, which was repeated four times at time intervals of 30 minutes $(0,30,60$, and $90 \mathrm{~min})$. Each reaction was carried out at room temperature with gentle stirring (10 revolutions per minute [rpm]) for a total of 2 hours. The doxorubicinconjugated phages were separated from the reaction by two dialysis steps against $1,000 \mathrm{~mL}$ of sterile $0.3 \mathrm{M} \mathrm{NaCl}$ each for 16 hours. Then, the doxorubicin-conjugated phages were precipitated using 4\% polyethylene glycol (PEG) (Bio Basic, Inc., Markham, ON, Canada) $/ 3 \% \mathrm{NaCl}$ on ice for 1 hour and were centrifuged at $9,000 \mathrm{rpm}, 4^{\circ} \mathrm{C}$, for 20 minutes. The supernatant was discarded and the pellet was resuspended with $1,000 \mu \mathrm{L}$ of phosphate Buffered Saline (PBS) (USB), pH 7.4.

\section{Evaluation of binding specificity of doxorubicin-carrying phages to soluble MICA antigen by enzyme- linked immunosorbent assay}

To evaluate the specific binding of doxorubicin-conjugated phages carrying anti-MICA, the reactions were tested as follows. After conjugation and dialysis, the solution from each $\mathrm{pH}(4,5$, and 6$)$ was precipitated with $4 \% \mathrm{PEG} / 3 \% \mathrm{NaCl}$ $(\mathrm{v} / \mathrm{v})$ on ice for 30 minutes and then centrifuged at 9,000 rpm, $4^{\circ} \mathrm{C}$ for 20 minutes. The supernatants and pellets of each were collected and tested for specific binding to sMICA antigen. A 96-well microtiter plate (Corning Incorporated Life Sciences, Tewksbury MA, USA) was treated with $300 \mu \mathrm{L}$ of carbonate buffer $\mathrm{pH} 9.6$ for 2 hours at room temperature. After discarding coating buffer, the sMICA antigens were coated on each well plate at 5 and $1 \mu \mathrm{g}$ in PBS (pH 7.4). The plate was wrapped with parafilm tape and incubated at $4{ }^{\circ} \mathrm{C}$ overnight. The solution was discarded, and the plate was then washed with phosphate buffer saline tween-20 (PBST) three times, followed by the addition of blocking solution, $300 \mu \mathrm{L}$ of 5\% skim milk (Becton, Dickinson and Company, Franklin Lakes, NJ, USA) PBST and incubated at room temperature for 2 hours. The blocking solution was discarded, and the plate was washed three times with PBST followed by the addition of $10^{11} \mathrm{PFU}$ of doxorubicin-conjugated phages in

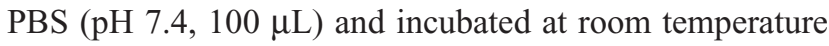
for 1 hour and the solution was then discarded. PBS ( $\mathrm{pH} 7.4)$ was used as a negative control. After washing three times with PBST, $100 \mu \mathrm{L}$ of anti-M13-conjugated horseradish peroxidase (HRP) (diluted at 1:15,000 in blocking solution; GE Healthcare Europe GmbH, Freiburg, Germany) was added to each well and incubated at room temperature for 1 hour. The antibody solution was discarded, and the plate was washed three times with PBST followed by the addition of $100 \mu \mathrm{L}$ of TMB substrate per well containing substrate A and substrate B at 1:1 (v/v) ratio (Kirkegaard and Perry Laboratories, Inc., Gaithersburg, MD, USA) and then incubated for 20 minutes in the dark at room temperature. After the blue color was developed, $50 \mu \mathrm{L}$ of stop reaction solution (2 $\mathrm{N} \mathrm{H}_{2} \mathrm{PO}_{4}$; BDH Laboratories Supplies, Poole, UK) was added to each well and yellow color was developed. The absorbance (optical density [OD]) at $450 \mathrm{~nm}$ was measured by the Sunrise enzyme-linked immunosorbent assay (ELISA) plate reader (Tecan, Grödig, Austria) and analyzed by the Magellen7 software (Tecan).

\section{Detection of doxorubicin molecules on phage particles \\ Detection of doxorubicin molecules on phage coat protein by $\mathrm{X}$-ray diffraction assay}

For the X-ray diffraction (XRD) assay, the samples were crystallized by lyophilizer (Martin Christ, Osterode am Harz, Germany) before analysis (provided by the Faculty of Dentistry, Khon Kaen University, Thailand). The samples, including doxorubicin $770 \mu \mathrm{g}$ in $1,000 \mu \mathrm{L}$ of distilled water, doxorubicin-conjugated phage $10^{12} \mathrm{PFU}$, doxorubicin and phage $10^{12} \mathrm{PFU}$ mixture in the solution of conjugation reaction with replaced EDC solution with distilled water and the naked phages $10^{12} \mathrm{PFU}$ in the same solution of conjugation reaction, were frozen at $-80^{\circ} \mathrm{C}$ overnight followed by frozen evaporation for 24 hours. All samples in crystallized form were analyzed by Philips X'Pert MPD X-ray diffractometer (Philips, Almelo, the Netherlands) using scanning angle $2 \theta$ (theta) ranging from $10 \theta$ to $70 \theta$, which were performed at and supported by the Department of Physics, Faculty of Sciences, Ubon Ratchathani University, Thailand. The spectrums from XRD were analyzed using the X'Pert data organizer software (Philips).

\section{Detection of doxorubicin molecules on phage coat protein by flow cytometry}

Doxorubicin is an autofluorescent agent, which can be excited and the emission detected by a fluorescent detector. ${ }^{20}$ The samples used in this experiment included two tubes of phages and doxorubicin-conjugated phages with $10^{7}$ phage particles. All samples were precipitated with $4 \% \mathrm{PEG} / 3 \% \mathrm{NaCl}$ on ice for 30 minutes and then centrifuged at $9,000 \mathrm{rpm}, 4^{\circ} \mathrm{C}$ for 
20 minutes. The supernatant was discarded, and the pellet was resuspended with $100 \mu \mathrm{L}$ of $\mathrm{PBS} \mathrm{pH}$ 7.4. The naked phages and doxorubicin-conjugated phages were stained with $2 \mu \mathrm{g} / \mathrm{mL}$ of anti-M13-conjugated FITC antibodies (Antibodies-online Inc, Atlanta, USA) and incubated on ice for 1 hour. Simultaneously, detection of doxorubicin molecules on phage coat proteins by the excitation at $488 \mathrm{~nm}$ with emission integrated above $530 \mathrm{~nm}$ and fluorescent signal of conjugated FITC anti-M13 was performed using the BD FACSCanto II Flow cytometer (Becton, Dickinson and Company). ${ }^{21-23}$ The raw data from all samples were analyzed by the BD FACSDiva software.

\section{Calculation of amount of doxorubicin on conjugated phages}

The standard curve of different concentrations of doxorubicin was performed to calculate the amount of doxorubicin on conjugated phages. The fluorescent intensity of doxorubicin was measured by Spectramax Gemini XPS fluorescence microplate reader (Molecular Devices LLC, Sunnyvale, CA, USA), using excitation at $485 \mathrm{~nm}$. The amount of doxorubicin tested was $20 \mu \mathrm{g}, 10 \mu \mathrm{g}, 5 \mu \mathrm{g}, 2.5 \mu \mathrm{g}, 1.25 \mu \mathrm{g}, 0.625 \mu \mathrm{g}$, $0.312 \mu \mathrm{g}, 0.156 \mu \mathrm{g}, 0.078 \mu \mathrm{g}, 0.039 \mu \mathrm{g}$, and $0.019 \mu \mathrm{g}$. This standard curve was used to estimate the amount of doxorubicin on conjugated phages.

\section{Evaluation of cytotoxicity of doxorubicin-conjugated phages by sulforhodamine $B$ assay}

The evaluation of cytotoxicity of doxorubicin-conjugated phages by SRB assay was modified from the protocol reported by Vichai and Kirtikara. ${ }^{24}$ In vitro cytotoxicity of doxorubicinconjugated phages was evaluated on Hela, KKU-M055, KKU-M213, KKU-M214, KKU-M139, and KKU-MMNK1, which are cancer cell lines expressing MICA antigens on their cell surface. Cells were harvested from an exponential phase culture by trypsinization with $1 \times$ trypsin/EDTA (Thermo Fisher Scientific, Waltham, MA, USA), counted and plated in 96-well plates in a triplicate manner. Optimal seeding densities for each cell line were determined to ensure exponential growth during 64 hours. Seeding densities were 4,500 cells per well. Thereafter, cells were incubated for 16-18 hours before exposure to testing samples.

Cell lines were treated in three conditions in triplicates. First, cell lines were treated with dilutions of free doxorubicin (1 $\mu \mathrm{g}, 0.5 \mu \mathrm{g}, 0.1 \mu \mathrm{g}, 0.05 \mu \mathrm{g}, 0.01 \mu \mathrm{g}, 0.005 \mu \mathrm{g}$, and $0.001 \mu \mathrm{g}$ ). Second, cell lines were treated with dilutions of each conjugated phages ranging from $10^{7}$ to $10^{12}$ particles as well as unconjugated or naked phages of $10^{12}$ particles. Third, cell lines were blocked with $10^{12}$ particles of anti-MICA-carrying phages with no drug conjugation 2 hours before Doxorubicin (Dox)-carrying phagedisplaying anti-MICA (Dox-phage) treatment. The amount of doxorubicin on phage particles are shown in Table 1. All testing samples were diluted in $100 \mu \mathrm{L}$ of complete Dulbecco's Modified Eagle's Medium and HAM's F12 medium. The treated cells were incubated in humidified incubator with $5 \% \mathrm{CO}_{2}$ at $37^{\circ} \mathrm{C}$ for 48 hours followed by the fixing step. The aside plate containing only cell suspension in three columns for no growth (day 0) was set up, and the plate was incubated at $37^{\circ} \mathrm{C}$ in a humidified incubator with $5 \% \mathrm{CO}_{2}$ until cell attachment completed and it was moved to the fixing step. In the fixing step, $100 \mu \mathrm{L}$ of cold $10 \%(\mathrm{w} / \mathrm{v})$ tricholo acetic acid (Sigma-Aldrich) was added to each well, and the plates were incubated at $4^{\circ} \mathrm{C}$ for 1 hour and then washed four times with slow tap water and the excess water was removed, followed by air-drying at room temperature. The cells were then stained with $100 \mu \mathrm{L}$ of $0.4 \%$ (w/v) SRB (Sigma-Aldrich, Munich, Germany) dissolved in 1\% (v/v) acetic acid (Merck, Germany) for 30 minutes and subsequently washed five times with $1 \%$ acetic acid to remove unbound stain. The plate was left to dry at room temperature, and bound protein stain was solubilized with $200 \mu \mathrm{L}$ of $10 \mathrm{mM}$ Tris base ( $\mathrm{pH} 10.5)$ per well and then shaken in the gyrator for 1 hour. The amount of SRB was measured for the absorbance at $492 \mathrm{~nm}$ using the ELISA plate reader(Tecan). The percentage of cell death was calculated using the formulae: $\left(\%\right.$ of cell death $=100-\left(\left[\right.\right.$ mean $\mathrm{OD}_{\text {sample }}-$ mean $\left.\mathrm{OD}_{\text {day } 0}\right] /\left[\right.$ mean $\mathrm{OD}_{\text {control }}-$ mean $\left.\left.\left.\mathrm{OD}_{\text {day } 0}\right]\right) \times 100\right) .{ }^{17}$ For $\mathrm{IC}_{50}$ determination, a dose-response curve was plotted between the sample concentrations and percent growth inhibition, which was derived from the $\mathrm{IC}_{50}$ calculation software (the CalcuSyn software; Biosoft Great Shelford, Cambridge, UK).

\section{Results \\ Specific binding of doxorubicin- carrying phages to sMICA antigens}

The specific binding of doxorubicin-conjugated phages displaying mutated anti-MICA antibodies (WW9B8.21) was evaluated by ELISA. The doxorubicin-conjugated phages

Table I The amount of doxorubicin on each dilution of Doxphage WW9B8.21

\begin{tabular}{ll}
\hline Dox-phage WW9B8.21 particles & Amount of doxorubicin $(\mu \mathrm{g})$ \\
\hline $10^{12}$ & 11 \\
$10^{11}$ & 1.1 \\
$10^{10}$ & 0.11 \\
$10^{9}$ & 0.011 \\
$10^{8}$ & 0.0011 \\
$10^{7}$ & 0.00011 \\
\hline
\end{tabular}

Abbreviation: Dox-phage, Dox-carrying phage-displaying anti-MICA. 
at $\mathrm{pH} 5$ showed the highest $\mathrm{OD}$ when compared to those derived from $\mathrm{pH} 4$ and 6 using the same EDC concentration (Figure 1). The results indicate that the process of doxorubicin conjugation did not destroy the antibody activities and that the activities against MICA were preserved. Most of conjugated phages were in the pellets after precipitation.

\section{Detection of doxorubicin molecules on phages by XRD assay}

The existence of doxorubicin molecules on phage particles was confirmed by two methods: the XRD assay and flow cytometry analysis. The spectrum patterns of free doxorubicin, naked phages, doxorubicin and phage mixture, and doxorubicinconjugated phages are shown in Figure 2. Doxorubicin revealed an amorphous spectrum, while the spectra of phages, doxorubicin-conjugated phages, and doxorubicin and phage mixture were shaped peaks. These spectra were similar; however, an additional peak was only found in the spectrum of doxorubicinconjugated phages (Figure 2), indicating that the spectrum of phage particles changed after doxorubicin conjugation.

\section{Detection of doxorubicin molecules on phage particles by flow cytometry}

The doxorubicin molecules are autofluorescent and can be detected by any fluorometer. Thus doxorubicin on phage particles could be excited and detected by the channel of peridinin-chlorophyll-protein complex by flow cytometer. With anti-M13 FITC staining, doxorubicin conjugated to phage particles could be indicated by double-fluorescent particles of peridinin-chlorophyll-protein complex and FITC. As shown in Figure 3, 98.8\% of phage particles were double fluorescent, indicating the existence of doxorubicin in phage particles. Using a doxorubicin standard curve (Figure 4), doxorubicin carried by phage solution used in the experiment could be calculated (Table 1).

\section{Amount of doxorubicin on conjugated phages}

The standard curve of different concentrations of doxorubicin and the equation for calculating the amount of doxorubicin on conjugated phages are shown in Figure 4. The fluorescence intensity of doxorubicin-conjugated phages at $10^{12} \mathrm{PFU}$ was 124.38. This standard curve was used to estimate the amount of doxorubicin on conjugated phages. The amount of doxorubicin was calculated using the formulae as follows:

$$
\begin{aligned}
Y(\text { fluorescence intensity })= & 10.83 X(\text { amount } \\
& \text { of doxorubicin })+5.256
\end{aligned}
$$

\section{Cytotoxicity of doxorubicin-conjugated phages on MICA-expressing cell lines}

The cytotoxic effect of doxorubicin-conjugated phages on MICA-expressing cell lines KKU-M214, KKU-M213,

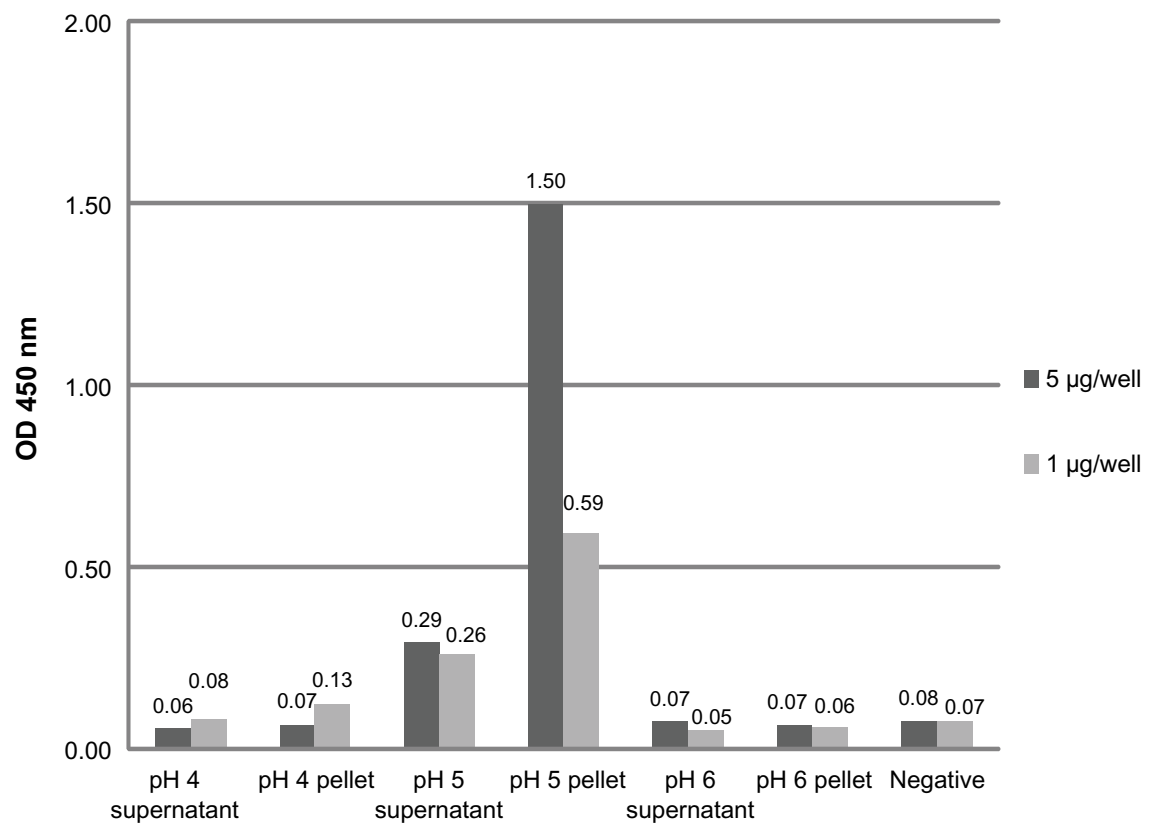

Figure I Determination of specific binding doxorubicin-conjugated phages at different $\mathrm{pH}$ conditions.

Notes: The doxorubicin-conjugated phages at $\mathrm{pH} \mathrm{4,5}$, and 6 were precipitated. Then, the supernatant and pellet were collected and tested for specific binding against sMICA antigen at $5 \mu \mathrm{g}$ and I $\mu \mathrm{g}$ per well. The pellet of phage conjugation at $\mathrm{pH} 5$ had the highest optical density indicating the best yield of doxorubicin-conjugated anti-MICA displayed phages.

Abbreviations: MICA, MHC class I chain-related A; sMICA, soluble MICA; OD, optimal density. 


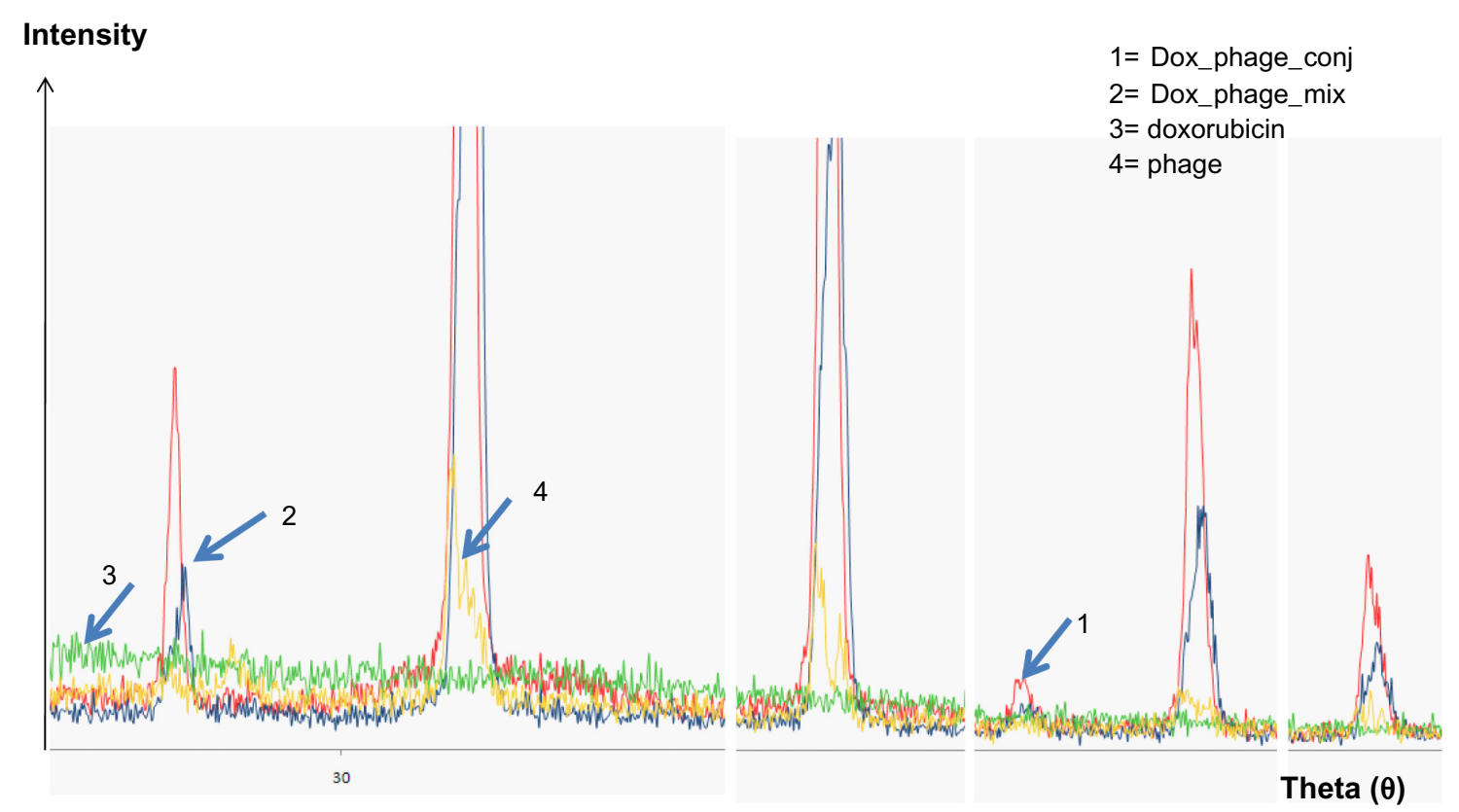

Figure 2 Magnified spectra of X-ray diffraction from crystallized samples of doxorubicin, phages, and conjugated phages.

Notes: Line I (red) represents doxorubicin-conjugated phages, and Line 2 (blue) represents doxorubicin and phage mixture. Line 3 (green) represents doxorubicin, and Line 4 (yellow) represents unconjugated or naked phages. This is the magnified spectra showing the changes in the magnitude of the spectra. An additional peak is evident in conjugated phages, suggesting a change in the structure.

Abbreviations: DOX_phage_conj, doxorubicin conjugated phages; DOX_phage_mix, mixture of doxorubicin and phages.

KKU-M055, KKU-M139, KKU-MMNK1, and Hela was determined by adding conjugated phages at different dilutions into the cells and comparing with doxorubicin-free drugs (Figure 5). The $\mathrm{IC}_{50}$ values were evaluated using the CalcuSyn demo version software and shown in Table 2 with the intensity of MICA expression on each cell line analyzed by flow cytometry. The doxorubicin-conjugated phages could kill the cell lines more effectively than free drug with a ratio of 0.8 to 6 (Table 2). In order to show the targeting effect, the MICA-blocking experiments were performed. Obviously, after blocking MICA by $\mathrm{mAb}$, the $\mathrm{IC}_{50}$ value of each cell line was increased and was comparable to that of free drug as shown in Table 2 and Figure 5 with the exception of KKU-M055, which is a doxorubicin-resistant cell line.

\section{Discussion}

Conventional chemotherapeutic agents are distributed nonspecifically in the body where they affect both cancer cells and
A

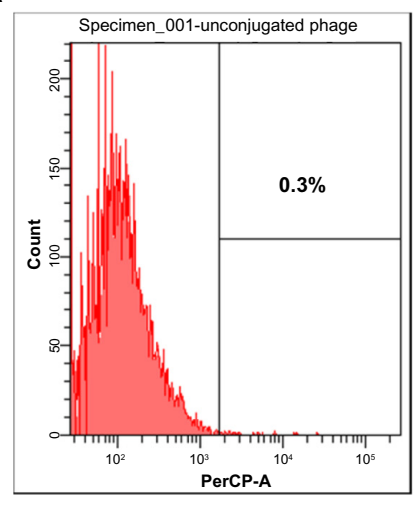

Unconjugated phage
B

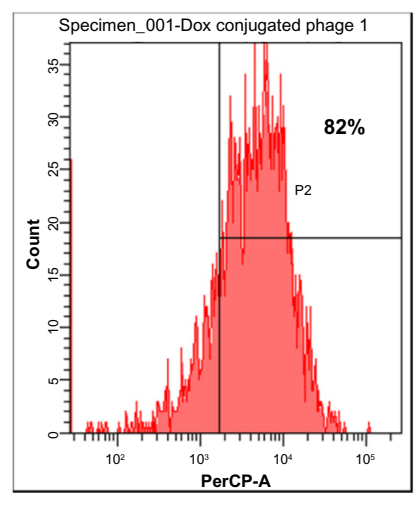

Dox unconjugated phage
C

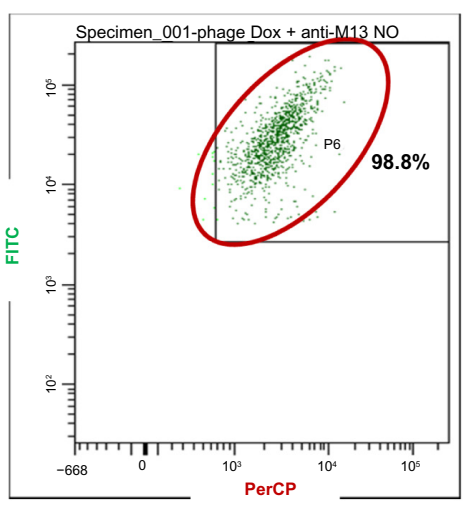

Double staining

Figure 3 Detection of doxorubicin molecules on phage particles by flow fluorometry.

Notes: Doxorubicin was detected in the peridinin-chlorophyll-protein complex channel by autofluorescence as shown in (A) naked phages and (B) doxorubicin-conjugated phages. Staining with FITC-conjugated anti-MI3 phages detected by both FITC and peridinin-chlorophyll-protein complex channels are shown in (C) indicating the conjugation of doxorubicin on phages.

Abbreviations: FITC, fluorescein isothiocyanate; Dox, Doxorubicin; PerCP, peridinin chlorophyll A protein. 


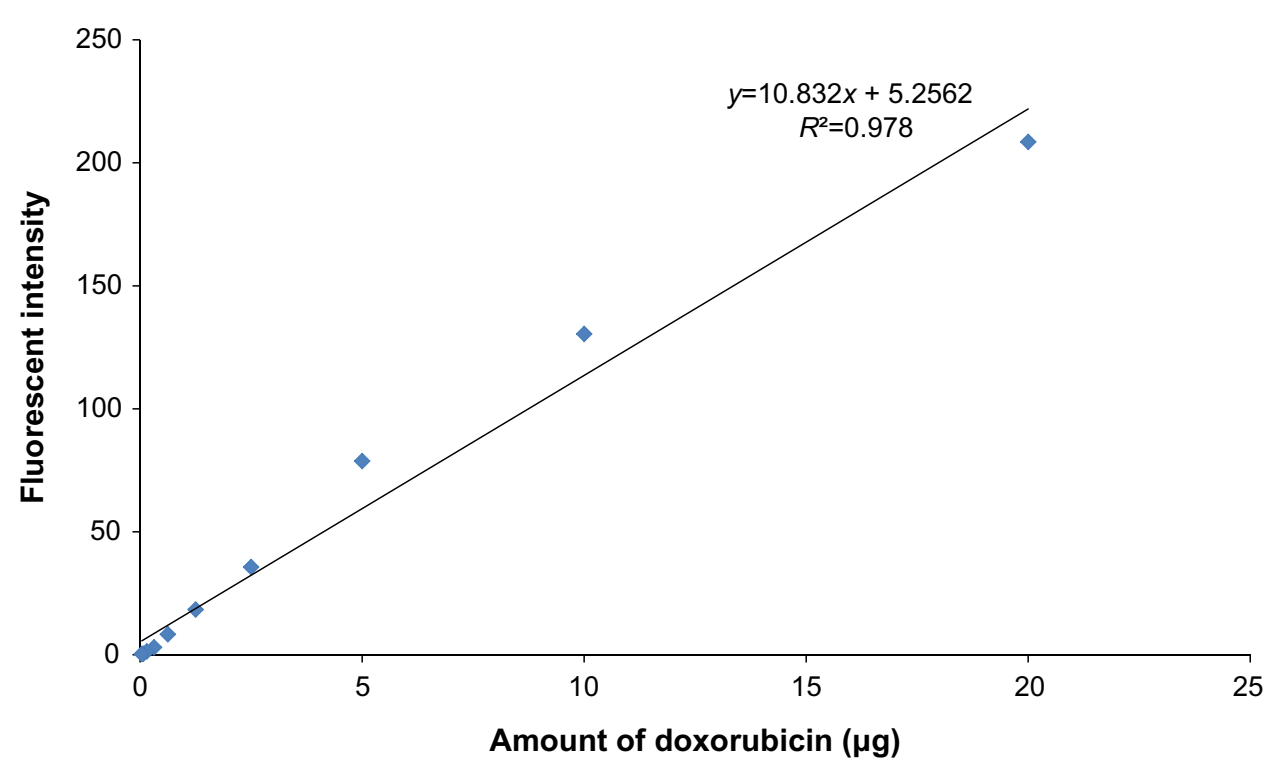

Figure 4 The standard curve of doxorubicin.

Notes: Doxorubicin of $20 \mu \mathrm{g}, 10 \mu \mathrm{g}, 5 \mu \mathrm{g}, 2.5 \mu \mathrm{g}, 1.25 \mu \mathrm{g}, 0.625 \mu \mathrm{g}, 0.312 \mu \mathrm{g}, 0.156 \mu \mathrm{g}, 0.078 \mu \mathrm{g}, 0.039 \mu \mathrm{g}$, and $0.019 \mu \mathrm{g}$ was used to generate a standard curve based upon fluorescent activities. This standard curve was used to estimate the amount of doxorubicin on conjugated phages.

normal cells. Molecularly targeted therapy has emerged as one approach to overcome the lack of specificity of conventional chemotherapeutic drugs. The mAbs that bind to specific markers on the surface of tumor cells offer an alternative therapy that is tumor specific and thus less toxic. The antibody-drug conjugates have been developed to increase the specific cytotoxicity on targeted tumor cells due to specific moieties and higher accumulated dose of anticancer drugs at targeted sites. ${ }^{25,26}$ However, this approach is limited by the amount of drug molecules that can be conjugated to antibody molecules. To overcome suboptimal dose, several nanoparticles are used as drug carriers using both passive and active targeting strategies that can enhance the accumulation of drug concentration at the tumor site while avoiding toxicity in normal tissues. ${ }^{1}$ Viral nanoparticles are virus-based nanoparticle formations ${ }^{27}$ that can be used for novel materials with a variety of properties including dynamics and self assembling systems. They can be produced in large quantities in a short time with programmable scaffolds. In addition, they are biocompatible and biodegradable with ability to display moiety for targeting.

Previously, the immunotherapy via NKG2D recognition has been established using chimeric antitumor $\mathrm{mAb} / \mathrm{NKG} 2 \mathrm{D}$ ligand complex ${ }^{28}$ which specifically bind to tumor cells and enhance the in vitro lysis by NK cells in an NKG2D-dependent manner as well as elicit adaptive immune responses against cancer cells independent of NKG2D ligand expression. ${ }^{14}$ Recently, a new form of targeted anticancer therapy has been generated in the form of a drug-carrying phage nanoparticles approach, which is based on genetically modified and chemically manipulated filamentous bacteriophages by Bar et al. ${ }^{3,4,17}$ The genetic manipulation endows the phages with the ability to display host specificity-conferring ligands. The phages are loaded with a large payload to a cytotoxic drug by chemical conjugation, which show that targeting of phage nanomedicines results in endocytosis, intracellular degradation, and drug release, resulting in growth inhibition of the target cells in vitro. ${ }^{4}$

In our study, MICA was used as a targeted moiety for the construction of anticancer-carrying filamentous phage nanoparticles. Doxorubicin was used as a therapeutic agent because it is widely used for treatment of many cancers. ${ }^{29}$ Conjugation of doxorubicin anticancer drug to phage $\mathrm{g} 8 \mathrm{p}$ coat proteins was performed using the EDC chemistry at mild acidic $\mathrm{pH}$ condition. The ratio of all reagents was optimized by calculation to gain the maximal drug conjugation. Previously, EDC, EDC-NHS, and EDC-sulfoNHS were employed to crosslink anti-HFA antibodies on APTES-functionalized platforms for immunodiagnostic applications. The results demonstrated more efficient antibody crosslinking by EDC in comparison to EDC-NHS and EDC-sulfoNHS at a normal $\mathrm{pH}$ of about 7.4, which increased the analytical performance and cost-effectiveness of immunoassays. ${ }^{30} \mathrm{In}$ addition, following NHS drug conjugation, the major coat g8p protein of phage particles was no longer recognized in ELISA by the commercial antiphage antibody. ${ }^{15}$

After conjugation reaction, detection of doxorubicin molecules on phage particles was performed by XRD assay and flow cytometry. The XRD assay is scanning of crystallized 


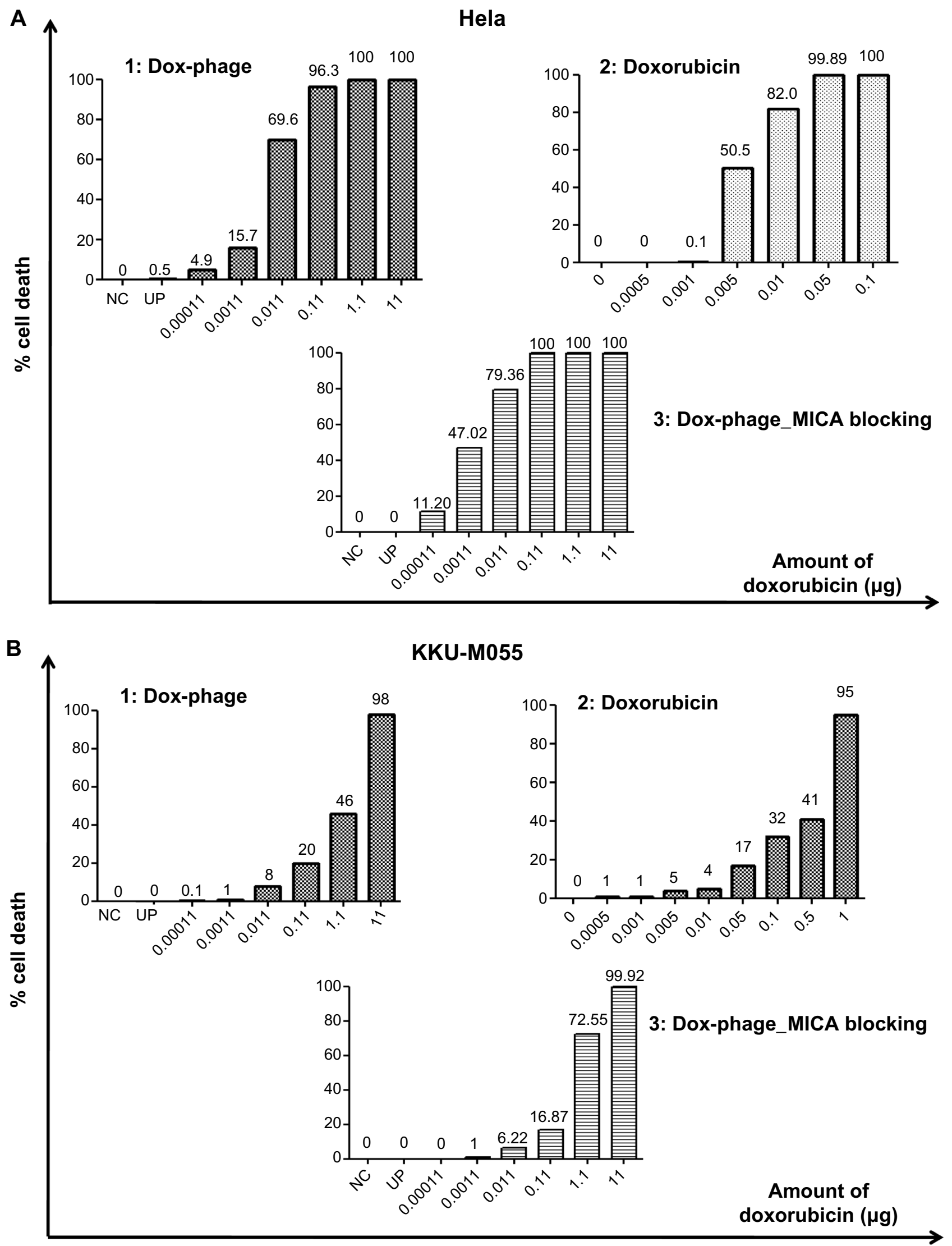

Figure 5 (Continued) 


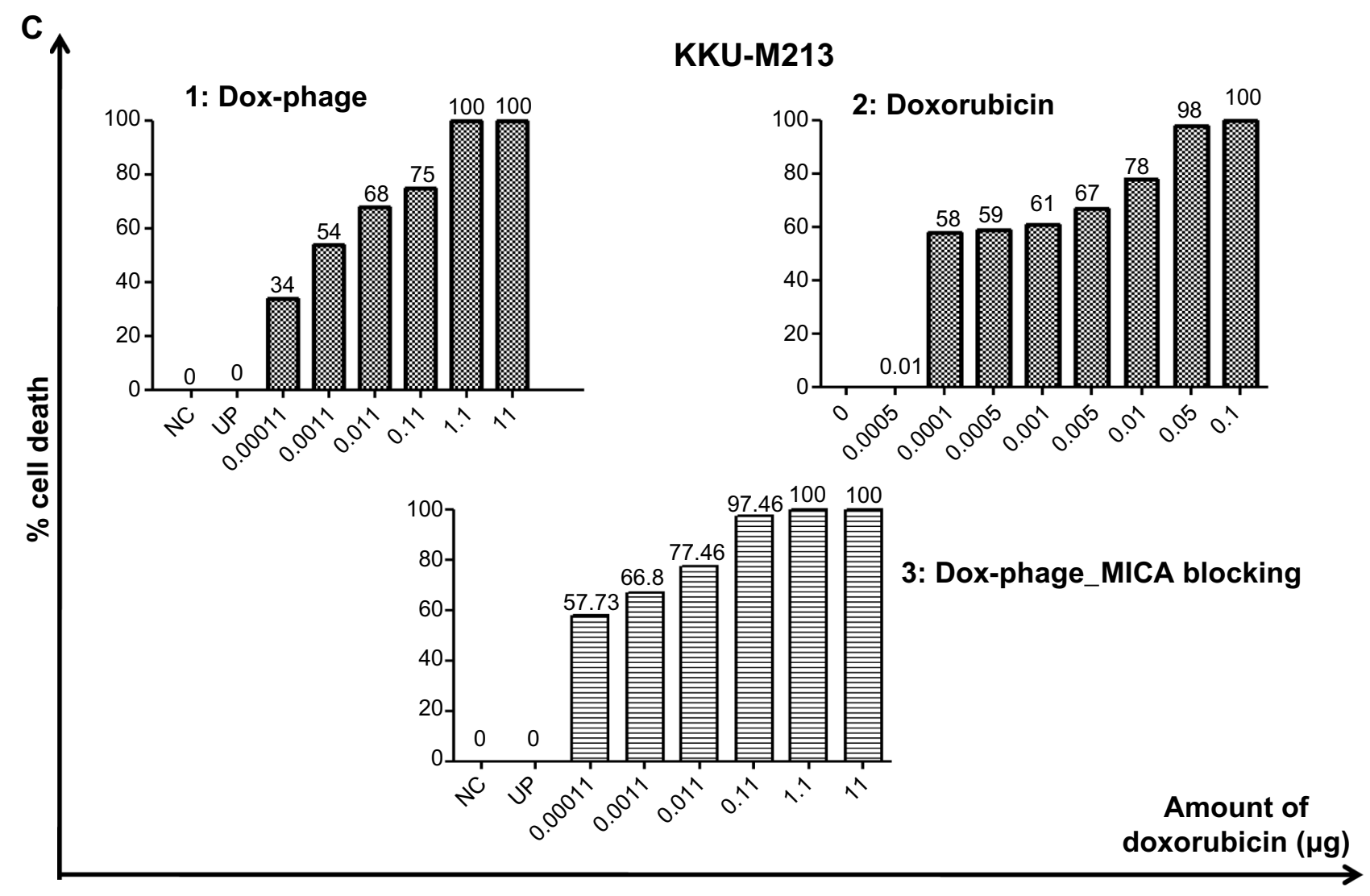

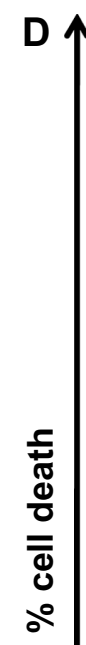

$D$


D
$\mathbb{0}$
$\frac{0}{0}$
$\overline{0}$
0
0

\section{KKU-M214}
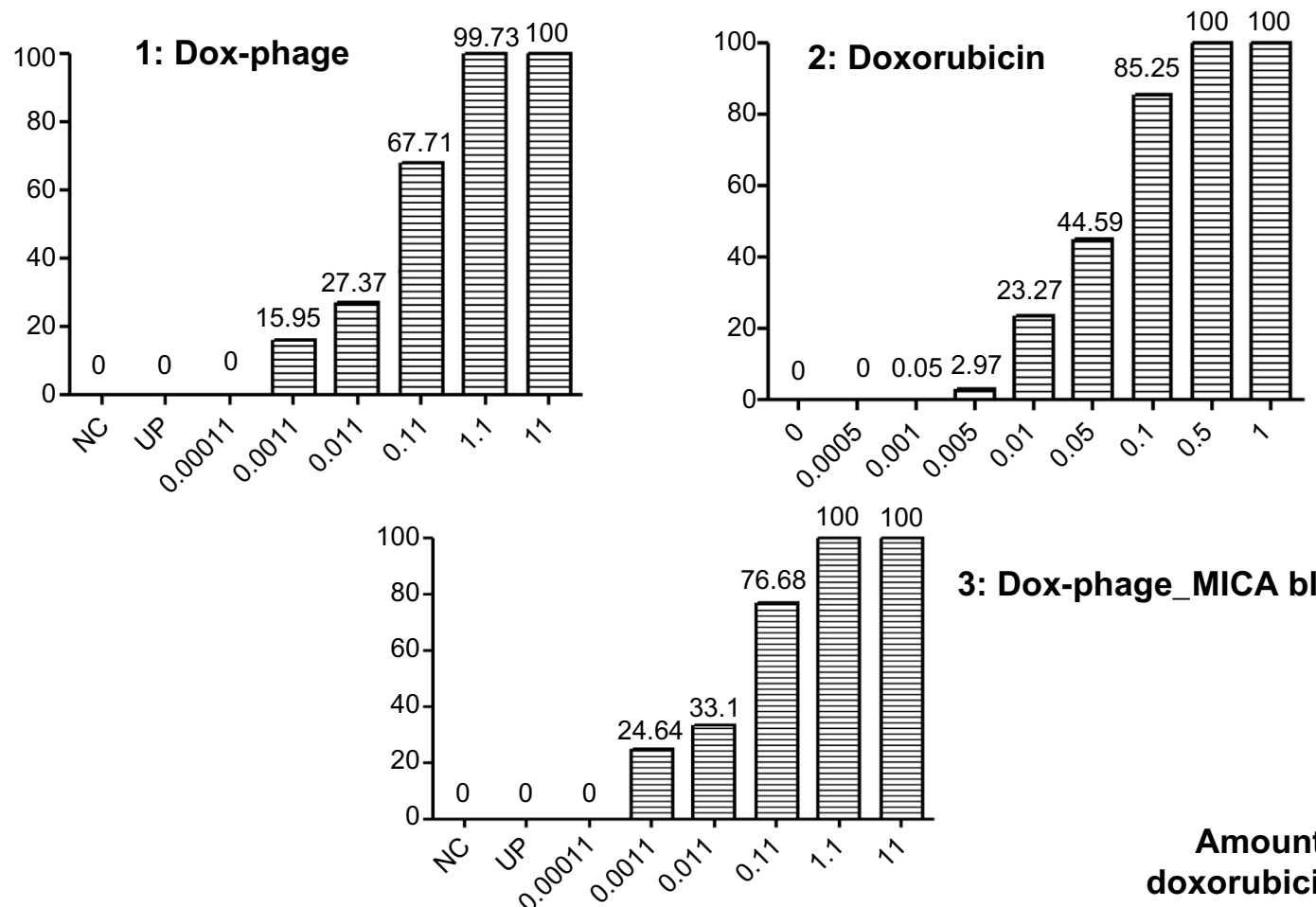

3: Dox-phage_MICA blocking

Amount of doxorubicin $(\mu \mathrm{g})$ 
E $\uparrow$
王
$\frac{\delta}{0}$
$\overline{\bar{\delta}}$
0
0

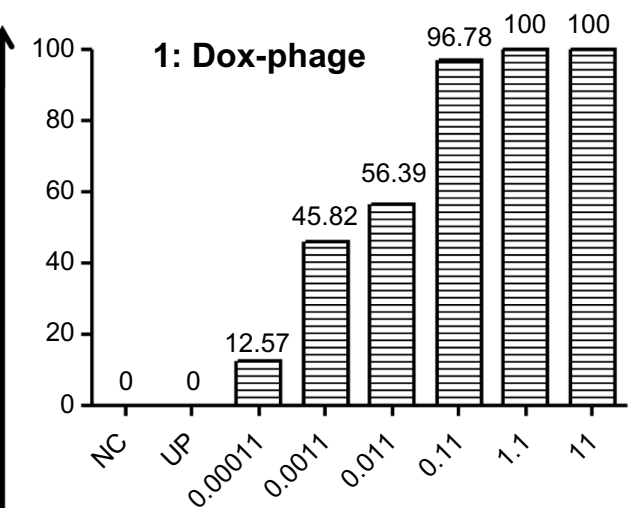

\section{KKU-M139}
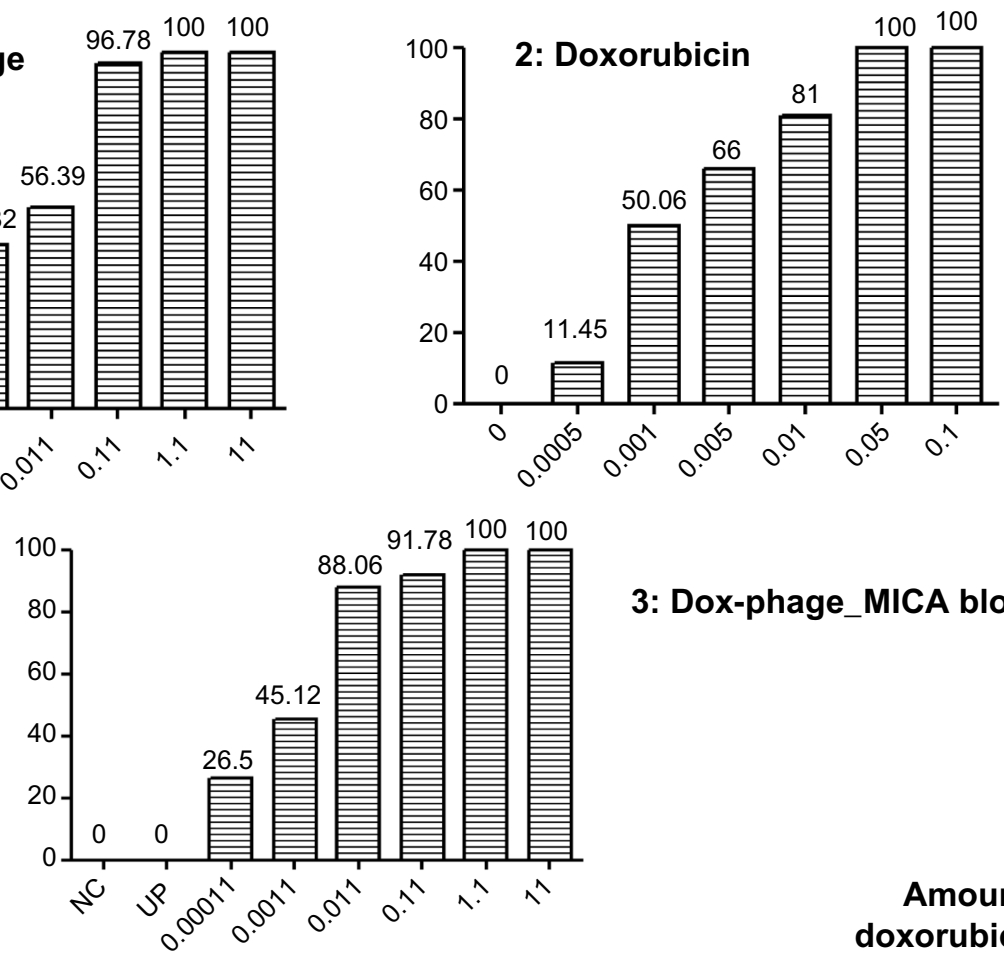

3: Dox-phage_MICA blocking

Amount of doxorubicin $(\mu \mathrm{g})$

\section{KKU-MMNK1}

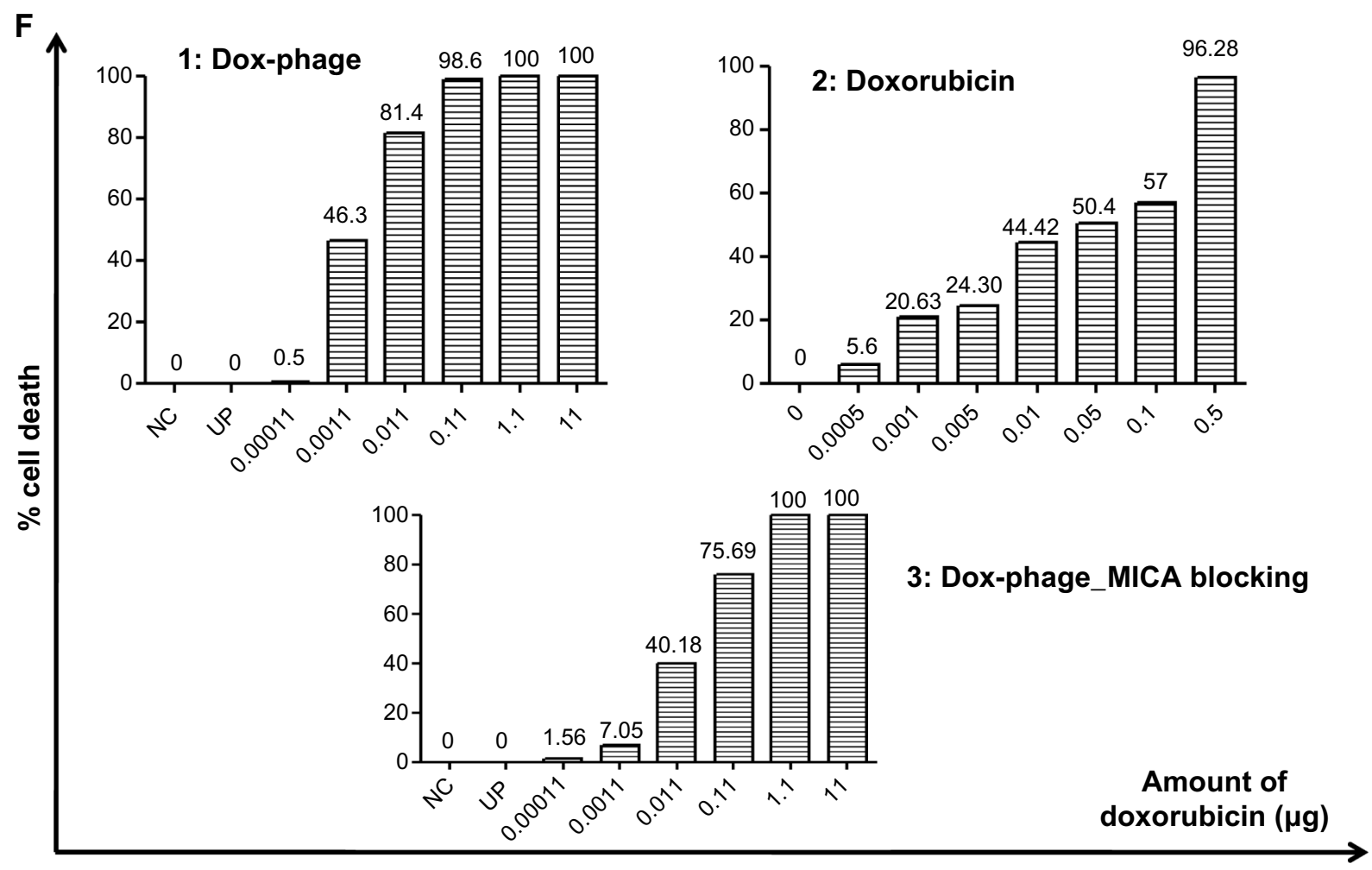

Figure 5 The toxicity effect of doxorubicin and Dox-phage mutant WW9B8.2I.

Notes: Percentages of cell death exposed to Dox-phages (I), doxorubicin (2), and DOX-phages after MICA blocking (3) are shown on (A) Hela, (B) KKU-M055, (C) KKUM2I3, (D) KKU-M2I4, (E) KKU-MI39, and (F) KKU-MMNKI. NC = negative control (HAM's FI2), UP = Unconjugated phages (I0'2 plaque-forming units).

Abbreviations: Dox-phage, Dox-carrying phage-displaying anti-MICA; MICA, major histocompatibility complex class I chain-related A. 
Table $2 I_{50}$ values of doxorubicin and doxorubicin-conjugated phages on MICA-expressing cell lines

\begin{tabular}{llllll}
\hline Cell lines & $\begin{array}{l}\text { IC } \\
\text { doxorubicin }\end{array}$ & $\begin{array}{l}\text { IC }_{50}(\mu \mathrm{M}) \\
\text { Dox-phage }\end{array}$ & $\begin{array}{l}\text { IC } \\
\text { after blocking MICA }\end{array}$ & $\begin{array}{l}\text { MFI of } \\
\text { MICA }\end{array}$ & $\begin{array}{l}\text { Ratio Dox- } \\
\text { phage }\end{array}$ \\
\hline KKU-M2I4* & 0.2896 & 0.1775 & 0.2327 & 5.77 & 1.6315 \\
KKU-M2I3* & 0.0103 & 0.0017 & 0.0086 & 4.05 & 6.0588 \\
KKU-M055* & 5.0000 & 5.3448 & 5.3328 & 1.29 & 0.7923 \\
KKU-MI39 & 0.0810 & 0.0258 & 0.0689 & 8.89 & 3.1395 \\
KKU-MMNKI & 0.3155 & 0.0948 & 0.2741 & 2.63 & 3.3280 \\
Hela** & 0.0830 & 0.0150 & 0.0793 & 25.00 & 5.5333
\end{tabular}

Notes: $I C_{50}$ was calculated by CalcuSyn Demo program. *Cholangiocarcinoma cell lines; **cervical cancer cell lines.

Abbreviations: Dox-phage, Dox-carrying phage-displaying anti-MICA; MFI, mean fluorescence intensity; MICA, major histocompatibility complex class I chain-related A; $\mathrm{IC}_{50}$, half maximal inhibitory concentration.

forms of particles by X-ray beam that is reflected by the crystallized particles. The reflected pattern can be observed as a spectrum. Particles with more crystallized forms show more shaped peak, while particles that are not crystallized or amorphous show a random spectrum. When particles have changed in structural conformation, different patterns can be observed when compared to those of original particles. The results indicated that doxorubicin-conjugated phages have structurally conformational changes after conjugation reaction. Additional shaped peak of doxorubicin-conjugated phages could be observed, suggesting the existence of doxorubicin molecules that were linked to phage amino acids. Higher intensity of doxorubicin-conjugated phages was observed indicating higher amount of conjugated doxorubicin (Figure 2). To ensure the drug conjugation on phages, flow cytometry was employed to confirm the Dox-phage conjugation. Flow cytometry was able to detect the signal of doxorubicin molecules due to the autofluorescent property, which can be excited and emitted. This has been successfully detected by flow cytometer in previous studies. ${ }^{21,22,31}$ The doxorubicin molecules can be triggered at $488 \mathrm{~nm}$ with emission integrated above $530 \mathrm{~nm}$ and can be detected by flow cytometer using the peridinin-chlorophyll-protein complex channel. To ensure that signal from doxorubicin was emitted from phage particles, second dye staining was required. The additional dye is anti-M13-conjugated FITC with emission at $530 \mathrm{~nm}$. This antibody is specific to $\mathrm{g} 8 \mathrm{p}$ coat proteins of phages. The samples of naked phages and doxorubicinconjugated phages stained with anti-M13-conjugated FITC were analyzed by flow cytometer. Conjugated doxorubicin can be simultaneously detected with the signal from FITC indicating the co-existence of doxorubicin and g8p. The percentage of phage particles conjugated with doxorubicin was about $82 \%$ with $98.8 \%$ of phages being double fluorescent (Figure 3). Detection of doxorubicin on conjugated phages that were stored at $4^{\circ} \mathrm{C}$ in PBS ( $\mathrm{pH}$ 7.4) for 1 month showed that the signal from doxorubicin was decreased by $10 \%$. This was presumed to be phage particles that had lost doxorubicin molecules during storage (data not show).

SRB assay was used to evaluate the cytotoxicity of doxorubicin-conjugated phages, Dox-phage WW9B8.21. Hela, KKU-M055, KKU-M213, KKU-M214, KKU-M139, and KKU-MMNK1 were treated in this experiment. These cells expressed MICA antigens at different levels indicated by mean fluorescence intensity. These cells were sensitive to doxorubicin-free drug with various $\mathrm{IC}_{50}$ values as shown in Table 2. The $\mathrm{IC}_{50}$ values of Dox-phages were two to six times lower than those of free drug, except for KKU-M055, which is more resistant to doxorubicin. Thus, Dox-phages have more killing efficiency than free drugs. In the case of KKU-M055, the use of free drug was not different from Dox-phages because of decreased MICA expression. However, the efficiency of killing is not only based on MICA expressions but also the drug-resistant factors carried by each cell line. The targeting effect of Dox-phages was indicated by the MICA-blocking experiments. $\mathrm{The} \mathrm{IC}_{50}$ values of Dox-phages when they were preblocked with anti-MICA were close to those of free drugs (Table 2). These results indicate that we have successfully developed the Dox-phages that are targeting MICA.

Conjugation of doxorubicin to phage $g 8 p$ coat proteins using EDC chemistry is one approach to construct the targeted therapeutic nanoparticles with no effect on binding moiety of antibodies on phage particles. The process of conjugation is easy and is done by adding EDC to the reaction at mild acidic $\mathrm{pH}$. However, amide bond between drugs and phage coat proteins is also sensitive to mild acidic $\mathrm{pH}$ as seen in the releasing experiment (data not shown). This is an advantage of these particles, which when reaching the tumor site with acidic $\mathrm{pH}$ environment were internalized into cells by endocytosis. Regarding immunogenicity of phage particles, antibacterial drug-carrying phages were evaluated in vivo, were shown to be nontoxic to mice and greatly reduced in immunogenicity and quickly cleared from the bloodstream. ${ }^{18}$ However, this has not been studied 
in humans. Thus, to avoid immunogenicity and other complications in humans, other nanoparticles such as dendrimer or liposome should be considered as a new platform of drug delivery system.

\section{Conclusion}

This study demonstrated the condition for conjugation of doxorubicin on phage particles displaying high-affinity antiMICA without affecting their MICA-binding activities. After conjugation, the doxorubicin molecules could be detected as conformational changes by XRD assay and double-fluorescent detection by flow cytometry. The linkages between conjugated doxorubicin molecules and phage proteins were sensitive to acidic $\mathrm{pH}$ and the affinity of targeted moiety also affected the cytotoxic activities on targeted cells. For further study, liposomes or dendrimers will be employed as new drug delivery platforms to avoid the problem of immunogenicity and safety in humans. The soluble Fab of anti-MICA antibodies will be produced and tagged on the surface of liposome/dendrimer that contains the anticancer drug. The new delivery system may be able to target selectively and show specificity to cancer cells overexpressing MICA.

\section{Acknowledgments}

This work was supported by the Higher Education Research Promotion and National Research University Project of Thailand, Office of the Higher Education, through the Center of Excellence in Specific Health Problems in Greater Mekong Subregion Cluster (SheP-GMS), Project no NRU 542022, and the Centre for Research and Development of Medical Diagnostic Laboratories, Khon Kaen University.

\section{Disclosure}

All authors have read and approved the manuscript and there are no conflicts of interest.

\section{References}

1. Sharkey RM, Goldenberg DM. Targeted therapy of cancer: new prospects for antibodies and immunoconjugates. CA Cancer J Clin. 2006;56: 226-243.

2. Wu AM, Senter PD. Arming antibodies: prospects and challenges for immunoconjugates. Nat Biotechnol. 2005;23(9):1137-1146.

3. Yacoby I, Benhar I. Targeted filamentous bacteriophages as therapeutic agents. Expert Opin Drug Deliv. 2008;5:321-329.

4. Bar H, Yacoby I, Benhar I. Killing cancer cells by targeted drug-carrying phage nanomedicines. BMC Biotechnol. 2008;8:37.

5. Pende D, Rivera P, Marcenaro S, et al. Major histocompatibility complex class I-related chain a and UL16-binding protein expression on tumor cell lines of different histotypes: analysis of tumor susceptibility to NKG2D-dependent natural killer cell cytotoxicity. Cancer Res. 2002;62:6178-6186.
6. Friese MA, Platten M, Lutz SZ, et al. MICA/NKG2D-mediated immunogene therapy of experimental gliomas. Cancer Res. 2003;63: 8996-9006.

7. Salih HR, Antropius H, Gieseke F, et al. Functional expression and release of ligands for the activating immunoreceptor NKG2D in leukemia. Blood. 2003;102:1389-1396.

8. Watson NF, Spendlove I, Madjd Z, et al. Expression of the stress-related MHC class I chain-related protein MICA is an indicator of good prognosis in colorectal cancer patients. Int J Cancer. 2006;118:1445-1452.

9. Groh V, Rhinehart R, Secrist H, Bauer S, Grabstein KH, Spies T. Broad tumor-associated expression and recognition by tumor-derived gamma delta T cells of MICA and MICB. Proc Natl Acad Sci U S A. 1999; 96(12):6879-6884.

10. Vetter CS, Groh V, Thor SP, et al. Expression of stress-induced MHC class I related chain molecules on human melanoma. J Invest Dermatol. 2002;118:600-605.

11. Melum E, Karlsen TH, Schrumpf E, et al. Cholangiocarcinoma in primary sclerosing cholangitis is associated with NKG2D polymorphisms. Hepatology. 2008;47(1):90-96.

12. Jinushi M, Takehara T, Tatsumi T, et al. Expression and role of MICA and MICB in human hepatocellular carcinomas and their regulation by retinoic acid. Int J Cancer. 2003;104:354-361.

13. Das H, Groh V, Kuijl C, et al. MICA engagement by human Vgamma2Vdelta2 $\mathrm{T}$ cells enhances their antigen-dependent effector function. Immunity. 2001;15(1):83-93.

14. Wodnar-Filipowicz A, Kalberer CP. Function of natural killer cells in immune defence against human leukaemia. Swiss Med Wkly. 2006; 136(23-24):359-364.

15. Phumyen A, Jumnainsong A, Leelayuwat C. Improved binding activity of antibodies against major histocompatibility complex class I chainrelated gene A by phage display technology for cancer-targeted therapy. J Biomed Biotechnol. 2012;2012:597647.

16. Wongsena W, Sconocchia G, Cho HS, et al. Production and characterization of monoclonal antibodies against major histocompatibility complex class I chain-related gene A. Tissue Antigens. 2008; 72(5):431-440.

17. Yacoby I, Shamis M, Bar H, Shabat D, Benhar I. Targeting antibacterial agents by using drug-carrying filamentous bacteriophages. Antimicrob Agents Chemother. 2006;50(6):2087-2097.

18. Yacoby I, Bar H, Benhar I. Targeted drug-carrying bacteriophages as antibacterial nanomedicines. Antimicrob Agents Chemother. 2007;51(6):2156-2163.

19. Nakajima N, Ikada Y. Mechanism of amide formation by carbodiimide for bioconjugation in aqueous media. Bioconjug Chem. 1995;6(1): 123-130.

20. Karukstis KK, Thompson EH, Whiles JA, Rosenfeld RJ. Deciphering the fluorescence signature of daunomycin and doxorubicin. Biophys Chem. 1998;73(3):249-263.

21. Krishan A. Monitoring of cellular resistance to cancer chemotherapy: drug retention and efflux. Methods Cell Biol. 2001;64:193-209.

22. Krishan A, Sauerteig A, Wellham LL. Flow cytometric studies on modulation of cellular adriamycin retention by phenothiazines. Cancer Res. 1985;45(3):1046-1051.

23. Krishan A. Flow cytometric monitoring of drug resistance in human tumor cells. Methods Cell Sci. 2002;24(1-3):55-60.

24. Vichai V, Kirtikara K. Sulforhodamine B colorimetric assay for cytotoxicity screening. Nat Protoc. 2006;1(3):1112-1116.

25. Ricart AD, Tolcher AW. Technology insight: cytotoxic drug immunoconjugates for cancer therapy. Nat Rev Clin Oncol. 2007;4:245-255.

26. Schrama D, Reisfeld RA, Becker JC. Antibody targeted drugs as cancer therapeutics. Nat Rev Drug Discov. 2006;5:147-159.

27. Yildiz I, Shukla S, Steinmetz NF. Applications of viral nanoparticles in medicine. Curr Opin Biotechnol. 2011;22:901-908.

28. Zhang T, Barber A, Sentman CL. Generation of antitumor responses by genetic modification of primary human T cells with a chimeric NKG2D receptor. Cancer Res. 2006;66(11):5927-5933. 
29. Tacar O, Sriamornsak P, Dass CR. Doxorubicin: an update on anticancer molecular action, toxicity and novel drug delivery systems. J Pharm Pharmacol. 2013;65(2):157-170.

30. Sandeep KV. Comparison of 1-Ethyl-3-(3-dimethylaminopropyl) carbodiimide based strategies to crosslink antibodies on amine-functionalized platforms for immunodiagnostic applications. Diagnostics. 2012;2(3): 23-33.
31. Krishna R, St-Louis M, Mayer LD. Increased intracellular drug accumulation and complete chemosensitization achieved in multidrug-resistant solid tumors by co-administering valspodar (PSC 833) with sterically stabilized liposomal doxorubicin. Int J Cancer. 2000;85(1):131-141.

\section{Publish your work in this journal}

OncoTargets and Therapy is an international, peer-reviewed, open access journal focusing on the pathological basis of all cancers, potential targets for therapy and treatment protocols employed to improve the management of cancer patients. The journal also focuses on the impact of management programs and new therapeutic agents and protocols on

\section{Dovepress}

patient perspectives such as quality of life, adherence and satisfaction. The manuscript management system is completely online and includes a very quick and fair peer-review system, which is all easy to use. Visit http://www.dovepress.com/testimonials.php to read real quotes from published authors.

Submit your manuscript here: http://www.dovepress.com/oncotargets-and-therapy-journal 